\title{
The Role of Noninvasive Measurement of Airway Inflammation in Asthma and Chronic Obstructive Pulmonary Disease
}

\author{
Joseph Mizrahi ${ }^{1}$ and Elizabeth Fireman² \\ 1. Sackler School of Medicine, Tel Aviv University, Tel Aviv, Israel; \\ 2. Laboratory of Pulmonary and Allergic Diseases, Tel Aviv Sourasky Medical Center, Tel Aviv, Israel
}

DOI: http://doi.org/10.17925/USRPD.2016.01.01.30

\begin{abstract}
Over the past few decades, a great emphasis has been put into developing and researching noninvasive techniques for monitoring respiratory functions in those with airway inflammatory diseases via biomarkers. The three most promising methods currently being researched are induced sputum analysis, exhaled nitric oxide measurement, and exhaled breath condensate analysis. Clinical applications of these three methods have the potential to transform the way we diagnose, monitor, and ultimately treat those with a variety of airway inflammatory diseases, such as asthma and chronic obstructive pulmonary disease (COPD). The rise in research into noninvasive airway biomonitoring is due to several factors. The ease of administering these tests compared with more invasive procedures, such as the bronchoalveolar lavage, enables physicians to more closely and effectively monitor airway inflammation in their patients, especially in vulnerable populations (children, aging, developing countries) that are not only more likely to suffer from these chronic respiratory diseases but also prove harder to perform invasive medical procedures on. In addition, there is an increasing goal of subclassifying different molecular and inflammatory phenotypes within these diseases, leading to more personalized therapies based on the specific composition of the inflammation. We present here the current understanding of biomarkers in these three techniques regarding asthma and COPD as well as the overall importance of determining airway inflammation composition.
\end{abstract}

\section{Keywords}

Noninvasive airway monitoring, airway inflammation, asthma, COPD, induced sputum, exhaled nitric oxide, exhaled breath condensate, vulnerable populations, multiple biomarker profile

Disclosure: Joseph Mizrahi and Elizabeth Fireman have nothing to disclose in relation to this manuscript. No funding was received in the publication of this article. Open Access: This article is published under the Creative Commons Attribution Noncommercial License, which permits any noncommercial use, distribution, adaptation, and reproduction provided the original author(s) and source are given appropriate credit.

Received: September 15, 2015 Accepted: October 9, 2015 Citation: US Respiratory \& Pulmonary Diseases, 2016;1(1):30-4

Correspondence: Joseph Mizrahi, Sackler School of Medicine, 17 East 62nd Street, New York, New York 10065, US. E: joseph1@mail.tau.ac.il

Airway inflammatory diseases are assessed and managed via multiple measurements, including airway caliber, airway responsiveness, and airway inflammation. Exacerbations post diagnosis is also a critical marker in terms of disease status and progression. Currently, diagnosis and monitoring of asthma ${ }^{1}$ and chronic obstructive pulmonary disease ${ }^{2}$ (COPD) is based largely on symptom reporting, pulmonary function tests, and bronchial reactivity. However, besides the inherent problems of subjective symptom reporting, it turns out that symptoms and lung function tests do not necessarily reflect the underlying airway inflammation in patients suffering from these diseases. ${ }^{3}$

While this association between asthma and COPD, and airway inflammation, has been well established, it is only recently that the actual composition of the inflammation (and not merely the presence of inflammation) has been studied in terms of determining the exact pathophysiology of these diseases, as well as to monitor disease progression and treatment. ${ }^{4}$ For example, with regard to asthma, the degree of eosinophilic airway inflammation has not only been shown to correlate with airway responsiveness, but in fact targeting airway eosinophilia specifically has consistently been associated with fewer asthmatic exacerbations, fewer hospitalizations, and fewer symptoms ${ }^{5,6}$ Moreover, the diagnosis of eosinophilic inflammation is more closely associated with a positive response to inhaled corticosteroids (ICS) than any other clinical measure, and thus the presence and degree of airway eosinophilia is critical in assessing one's possible response to ICS as well as altering ICS doses once treatment begins. ${ }^{4}$

Patients that suffer from COPD would also benefit immensely from the determination of their airway inflammation composition. It has been shown that those with elevated levels of C-reactive peptide (CRP), fibrinogen, and leukocytes in their lower airways are more likely to suffer from COPD exacerbations. ${ }^{7}$ Asthma and COPD can coexist as part of an "overlap syndrome," and asthma can even be a risk factor for developing 
COPD in the future, making distinguishing between these two related but distinct diseases via determination of airway inflammation critical in providing appropriate care. ${ }^{2}$

These are but a few of the discoveries made using airway inflammation to monitor and treat asthma and COPD and that represent the enormous potential that monitoring of airway inflammation can have on the way physicians approach these diseases. This article will further elucidate on the importance of determining airway inflammation composition as well as review some of the known biomarkers currently being studied to monitor asthma and COPD.

\section{Review}

\section{Airway Inflammatory Phenotypes}

Determining the exact composition of airway inflammation has become extremely important in classifying the different subtypes of asthma and COPD, which will enable physicians to better cater therapy for their patients. While eosinophilic asthma has been well studied and treatments have been developed that specifically target airway eosinophilia, it is well established that there are those that fit the clinical definition of asthma without an associated increase in their eosinophil count. In fact, there have been four phenotypes of asthma identified based on the inflammatory cell composition of the subject's lower airways found via induced sputum (IS): eosinophilic ( $\geq 3 \%$ ), neutrophilic ( $\geq 61 \%$ ), mixed type ( $\geq 3 \%$ eosinophils and $\geq 61 \%$ neutrophils), and paucigranulocytic type $(<3$ $\%$ eosinophils and $<61 \%$ neutrophils). ${ }^{3}$ Knowing the exact composition of inflammatory cells and correlating it with symptoms and pulmonary function tests will lead to more research into how to best treat each asthma subtype and improve overall care.

It is also important to note that while cellular phenotypes have been shown to stay consistent over time in asthmatic adults, ${ }^{8}$ the same cannot be said for children, whose cellular asthmatic phenotype can change from noneosinophilic to eosinophilic and vice versa. ${ }^{9}$ Therefore, being able to easily monitor airway inflammation alterations in children is vital to providing the appropriate care and treatment that they need.

There is also work being done to determine different COPD phenotypes. Efforts have been made to do this using computer tomography, ${ }^{10}$ symptoms and exacerbations, ${ }^{11}$ comorbidities, and lung function tests. ${ }^{12}$ Despite all being valid, if physicians truly wish to understand and subsequently tailor treatment toward different COPD phenotypes, then assessing airway inflammation, rather than using other measurements, represents the most accurate way of subclassifying the heterogeneous disease.

\section{Burden on Vulnerable Populations}

While the health of the world is by most accounts rising, more and more people are developing chronic conditions due to fewer dying of more acute and communicable diseases. This is especially pertinent regarding chronic respiratory diseases, such as asthma and COPD. Millions of people across the globe suffer from these chronic airway diseases, and the prevalence of these chronic conditions has only been growing worse. Between 1990 and 2013, the prevalence of asthma increased by $32.10 \%$, while the prevalence of COPD rose by a staggering $65.13 \%$. COPD is also ranked as the eighth leading cause of years lived with disability (YLD) in the world. ${ }^{13}$
The burden on developing countries from chronic airway diseases is particularly immense due to a multitude of factors, including a lack of basic infrastructure for dealing with chronic diseases (due to the focus on communicable diseases), high exposure rates to chronic airway disease risk factors (indoor pollution, smoking, use of solid biomass fuels), and a lack of appropriate diagnostic tests as well as drugs. ${ }^{14}$ Also, in addition to the direct medical costs that these diseases inflict, the loss of human capital is detrimental to a developing country's young economy. Since many of these chronically ill patients cannot work and need another person to help them in their daily living, the indirect costs of asthma and COPD can pose a grave threat to their economies. ${ }^{2}$ Indeed, COPD and asthma rank 4 and 14, respectively, in terms of disability-adjusted life years (DALYs) at the global level (COPD—29.8 DALYs, Asthma-13.9 DALYS). ${ }^{15}$ Therefore, it is in the best interest, both medically and economically, to be able to easily and accurately diagnose and monitor those that suffer from these chronic diseases at a low cost.

In addition to people living in developing countries, those living at either end of the age spectrum will also gain from improvement of respiratory monitoring, as both children and the aging have a growing prevalence of chronic airway diseases. Childhood asthma is responsible for many lost school days, leading to deficits in their social and academic development, particularly in lower socioeconomic communities. ${ }^{16}$ While those that are actually diagnosed with asthma will inevitably require more resources, those that live in lower income countries tend to be underdiagnosed, leading to more emergency room visits for their symptoms and multiple complications, which inevitably lead to higher costs of care in these countries. ${ }^{17,18}$

Regarding aging, it is no secret that the world's population is getting older, with life expectancy increasing and noncommunicable diseases becoming the primary cause of death in both developed and developing countries. ${ }^{19}$ The financial and medical burden that this phenomenon will have on the world is enormous, and medicine must attempt to catch up. That means we need to not only be able to diagnose chronic conditions early in their disease course but be able to monitor and treat these patients efficiently and cost-effectively. While asthma for the most part affects younger populations, COPD is generally found in those 40 and older. Classically underdiagnosed, the prevalence of COPD in those above the age of 40 ranges from $4 \%$ to $20 \%$ globally and will only rise as the world continues to age. By 2030, COPD will rank fourth in the leading causes of death in the world and seventh in terms of worldwide burden of disease. ${ }^{20}$

\section{Noninvasive Techniques for Monitoring Airway Inflammation}

Once the importance of determining and monitoring airway inflammation is well understood and accepted, the question that inevitably arises is how to best accomplish this. Bronchoscopy with airway wall biopsies and bronchioalveolar lavage (BAL) have been considered the gold standards to determine airway inflammation. However, these procedures are extremely invasive and require highly trained specialists to induce anesthesia, perform the procedures, and analyze the results. ${ }^{21}$ In addition, the complexity of these procedures requires the cooperation of the patient, which is sometimes difficult to achieve, especially with children.22

In order to allow assessment of airway inflammation to become a standard aspect of clinical practice, noninvasive techniques must be developed 
that are both easy for the physician or technician to perform and the subjects themselves to receive. The tests obviously must be reproducible and valid, and the cost of the overall procedure and analysis must be low enough to be adopted in low resource areas. There are three noninvasive procedures currently being used or studied that show the most promise in terms of developing reliable measurements of airway inflammation, and with further research they will hopefully replace invasive airway monitoring altogether instead of merely being a surrogate to biopsy and $\mathrm{BAL}$ results as is currently their use.

\section{Induced Sputum Analysis}

Currently, IS analysis is the most widely used technique to noninvasively determine airway inflammation. Due to sputum containing secretions from the tracheobronchial tree rather than from the upper airways, inflammatory cells found in the sputum sample can be indicative of a lower respiratory airway disease, such as asthma or COPD.23

While sputum can be obtained either spontaneously or by inducement, IS via inhaling a concentration of nebulized hypertonic saline solution has been shown to contain a higher proportion of viable cells, less squamous cell contamination, and better-quality cytospins and is thus preferred over spontaneous sputum collection. ${ }^{24}$ The subject is encouraged to cough and expectorate a sputum sample into a plastic vial, which is then processed to generate differential cell counts. This process has been shown to be highly reproducible and valid in a number of studies. ${ }^{25,26}$ In addition, there is a strong correlation between the cellular composition of airway inflammation found using BAL and IS, making IS the preferred mode of monitoring airway inflammation due to its less invasive nature when a BAL would otherwise be indicated. ${ }^{27}$

IS can be of immense value to the clinical physician, especially in cases when standard methods of diagnosis (history, spirometry, physical examination) fail to yield an explanation for unclear cough, such as in cough-variant asthma (CVA). A differential cell count can be obtained, and the presence and level of eosinophilic inflammation, for example, in asthmatics, can be used as an indication for ICS or for the adjustment of asthma therapy. ${ }^{28}$ Moreover, determining if one's airway inflammation is noneosinophilic is also important, as neutrophilic inflammation is more indicative of COPD than asthma and is associated with increased Global Initiative for Chronic Obstructive Lung Disease (GOLD) stage as well. There are ongoing studies using IS analysis that can assess COPD severity, progression, and risk for future exacerbations. ${ }^{29}$ Finally, the classification of asthma and COPD phenotypes based on IS cell counts will further our understanding of these diseases and will aid in future research.

In addition, IS can serve as a biological monitoring method for those that are exposed to airborne particulate matter (PM). PM is implicated in a host of lung problems and can be found in a variety of occupational exposures and in outdoor pollution. Is has been shown to be able to detect PM in welders, ${ }^{30}$ foundry workers, ${ }^{31}$ and even in firefighters who were exposed to dangerous dust particles after the collapse of the World Trade Center years after the exposure.32 Outdoor pollution has been proved to exacerbate asthma in children, ${ }^{33}$ and IS has been successfully used to detect PM in urban children, even correlating with the presence of eosinophilic inflammation in these patients. ${ }^{34}$ Overall, the ability of IS to detect PM in airways is another clinically useful benefit of the procedure, especially in vulnerable populations that are exposed to higher percentages of PM.

While IS is the most studied and widely implemented of the noninvasive techniques, it does have its drawbacks. It is to some extent invasive, albeit much less so than BAL. IS has proved difficult to perform in children and very ill patients, as the act of expectorating sputum can be stressful for these subjects and some even experience bronchospasm after inhaling the hypertonic saline. ${ }^{35}$ Performing the procedure, as well as analyzing the sputum samples, requires highly trained lab technicians, limiting the widespread adoption of this method in locations lacking these resources and thus only making IS available in specialized medical centers. Despite efforts to develop shorter and simpler processing of IS samples, ${ }^{36}$ these factors as a whole currently limit IS from becoming a standard part of asthma and COPD maintenance.

\section{Exhaled Nitric Oxide Measurement}

Another biomarker that can be used to asses airway inflammation is exhaled nitric oxide (FeNO). In the lungs, nitric oxide (NO) is produced by the enzyme NO synthase, which is upregulated in different states of inflammation. ${ }^{37}$ Indeed, the association between airway inflammation and increased levels of FeNO in breath has been known and appreciated for the past few decades. ${ }^{38}$

There are several methods of collecting FeNO from patients, but they are generally divided into online and offline collections. While online measurement entails real-time analysis of NO content in exhaled breath via a computer, offline measurement involves the collection of exhaled gas in a reservoir and analysis of the NO content at a later time. Both have their advantages and disadvantages, and recent efforts have been made to standardize FeNO collection in order to obtain accurate benchmarks for measurements of FeNO. ${ }^{39}$

Using FeNO measurements to manage and monitor asthma and COPD is an exciting prospect that has undergone much study. Increased FeNO levels have been shown to correlate with eosinophilic inflammation in both asthmatic adults ${ }^{40}$ and children, ${ }^{23}$ and a reduction in FeNO levels has been found in patients with asthma that have clinically improved with ICS treatment. ${ }^{41}$ Asthma symptoms, bronchial hyperresponsiveness, and abnormal lung function have all been found to correlate with FeNO levels as well. ${ }^{42}$ COPD patients with increased levels of FeNO have been found to respond positively to salbutamol treatment, ${ }^{43}$ and there is ongoing research into whether FeNO can be monitored as an indicator of treatment response to ICS in patients with COPD. ${ }^{44}$ Practically speaking, the relative ease of FeNO collection, both in terms of administering the test and for the patients themselves (especially children), as well as the immediacy of the results (for online collections $\sim 5$ minutes), makes FeNO an attractive option for noninvasive analysis of airway inflammation.

Despite its advantages, there are numerous problems and limitations of FeNO that would restrict the procedure from becoming clinically used on a daily basis. First and foremost, although there have been numerous studies showing the usefulness of measuring FeNO, many have used different cutoffs and thus a standardized approach to measuring FeNO is needed in order to further prove its benefit. High levels of FeNO are not specific or sensitive for asthma or COPD, but rather simply imply 
inflammation of the airways. ${ }^{23}$ The cost of purchasing a chemiluminescence analyzer to measure and analyze FeNO is extremely high, compared with IS collection or exhaled breath condensate (EBC) analysis. However, the biggest obstacle facing FeNO adoption is that its levels are affected by the specific atopic, smoking, and ICS usage status of the patient, and thus determining a baseline benchmark for "normal" FeNO levels remains elusive. While there has been development of non-chemiluminescencebased analyzers, such as the NIOX MINO ${ }^{\circledR}$ or NIOX VERO ${ }^{\circledR}$ (Aerocrine, Solna, Sweden), which would bring the cost down, there are still significant disadvantages to using FeNO and it therefore remains a research tool rather than a clinically useful one for the time being. ${ }^{29}$

\section{Exhaled Breath Condensate}

Perhaps the most innovative and intriguing method of noninvasive airway inflammation measurement is analysis of EBC. It is obtained easily by tidal breathing into a special mouthpiece for 10 minutes. The exhaled breath is then cooled in a Teflon tube or double-glass layer container into droplets to be later analyzed. ${ }^{45}$

The scientific understanding of airway inflammatory diseases has evolved over the years, recognizing that there are several concurrent mechanisms underlying the pathophysiologies of these diseases rather than a single identifiable cause. While IS can obtain differential cell counts and FeNO can determine exhaled NO, EBC can measure a whole host of biomarkers in exhaled breath creating a multiple biomarker profile of a specific subject's airway inflammation. A reduction in EBC pH levels has been the most studied and reproducible finding in patients with asthma and COPD. ${ }^{46}$ Other EBC biomarkers that indicate airway inflammation have been identified, including an increase in $\mathrm{H}_{2} \mathrm{O}_{2}$, leukotrienes, interleukins, prostaglandins, and 8-isoprostane. ${ }^{47}$

Due to this ability to simultaneously measure several biomarkers in exhaled breath, EBC shows the most promise of the three discussed techniques in terms of aiding research into subclassifying different phenotypes of asthma and COPD. The EBC multiple biomarker panel can also potentially assess the severity of the airway inflammation itself, monitor ongoing treatment, and predict exacerbations early in the disease course. ${ }^{44} \mathrm{EBC}$ has proved useful in detecting ultrafine particles (UFP $<0.1 \mu \mathrm{m})$ in asthmatic children, correlating well with wheezing, breath symptom score, and IS eosinophilia. ${ }^{88}$ Moreover, EBC has been shown to be an easy and agreeable means of determining airway inflammation in both children with asthma ${ }^{49}$ and elderly COPD patients. ${ }^{50}$

The shortfalls of EBC are similar to those of FeNO, with the high cost of machines needed for the procedure (though still cheaper than FeNO measurement), as well as the various confounding factors (environmental contaminants, age, gender, nutrition, ongoing medications, smoking) that would alter the results in individual patients. The lack of standardization and reference values also pose a problem for introducing EBC into regular clinical use. ${ }^{51}$ Finally, the need for specialized labs to interpret the results will impede EBC from being adopted in low resource areas..$^{50}$

\section{Conclusion}

While the three discussed techniques represent the most studied and well-researched noninvasive means of determining airway inflammation, there are other recently discovered biomarkers that indeed warrant

\section{Table 1: Advantages and Disadvantages of Three Noninvasive Methods of Determining Airway Inflammation}

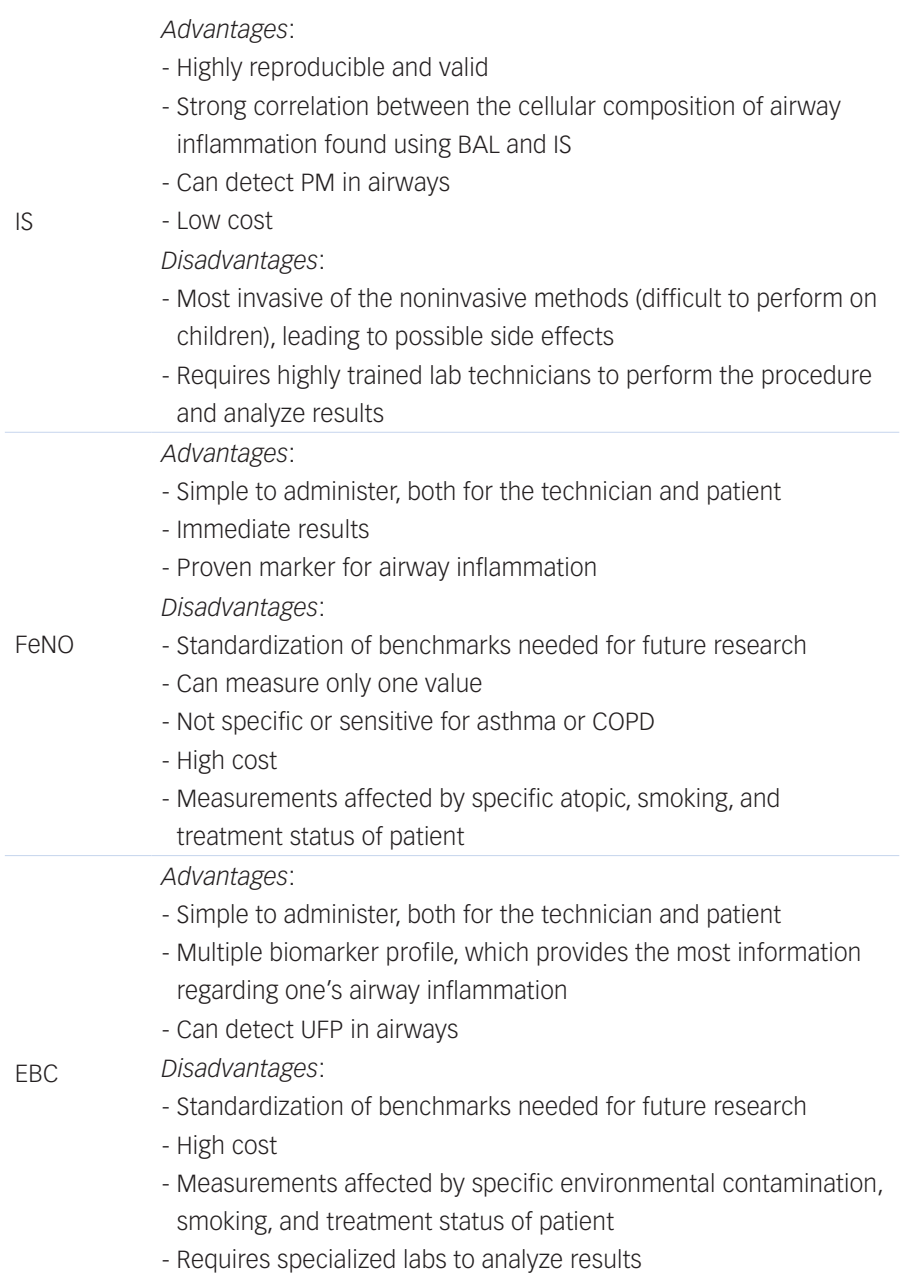

$B A L=$ bronchoalveolar lavage; $C O P D=$ chronic obstructive pulmonary disease; $E B C=$ exhaled breath condensate; FeNO = exhaled nitric oxide; IS = induced sputum; $P M=$ particulate matter; UFP $=$ ultrafine particles

further investigation. Serum periostin, which is secreted by airway epithelial cells in response to inflammation (IL-13 specifically), has been shown to correlate strongly with airway eosinophilia better than any other serum measurement. ${ }^{52}$ Several genetic loci have been identified that are associated with asthma risk, including ORMDL3, ADAM33, and several cytokine and cytokine-receptor genes..$^{53}$ Finally, the study of metabolomics is emerging as an innovative way to differentiate between different chronic airway inflammatory phenotypes. The pattern of molecules generated from cellular metabolic activities in these patients is distinct depending on the particular pathophysiology of the underlying disease. Analysis of these different "metabolomic profiles" or "breath prints" using spectroscopy or the electric nose (which has a host of nanosensors that can detect these different molecules and the patterns they make) represents yet another promising noninvasive method of determining airway inflammation. ${ }^{54}$

Presently, the prospect of using a single biomarker in order to assess asthma and COPD airway inflammation is becoming increasingly invalid and obsolete, with a multiple biomarker profile emerging as a more 
clinically useful tool. ${ }^{3}$ This is due to the ever-increasing understanding that airway inflammatory diseases are heterogenous, with multiple phenotypes and multiple underlying mechanisms of disease being present in both asthma and COPD. Thus, future study should be targeted toward combining the results of different airway inflammatory measurements, providing a more comprehensive analysis of the subject's airway inflammation. Currently, there are several research groups, such as the Severe Asthma Research Network (SARP) ${ }^{55}$ and Unbiased BIOmarkers in PREDiction of respiratory disease outcomes (U-BIOPRED), ${ }^{56}$ which are exploring this avenue of thought.

There has been an explosion of research into noninvasive airway monitoring over the past couple of decades, as airway inflammation is increasingly being understood as central to the pathogenesis of chronic airway diseases. Determining different phenotypes within these diseases is also becoming vital to providing appropriate and individualized care. While invasive procedures, such as biopsies and BAL, have provided a means of determining the composition of airway inflammation in the past and still represent the most useful techniques in terms of yielding pathophysiological criteria for these diseases, noninvasive techniques must be further developed and studied in order to address the rising prevalence of these diseases in children and the aging, particularly in an economically feasible way in order to address those living in low resource countries. While IS and FeNO are attractive options, EBC presents the best hope of introducing monitoring of airway inflammation into clinical practice, due to its ability to create a multiple biomarker profile, its relative affordability, and the ease of administering and receiving the procedure (see Table 1). More research into these procedures will inevitably transform our understanding and management of chronic airway inflammatory diseases in the future.
1. GINA: Global Strategy for Asthma Management and Prevention (Updated 2015), Bethesda, MD: National Institutes of Health, National Heart, Lung, and Blood Institute, 2015.

2. Global strategy for the diagnosis, management, and prevention of chronic obstructive pulmonary disease: Revised 2015. Global Initiative for Chronic Obstructive Lung Disease (GOLD). Available at: www.goldcopd.org (accessed on September 1, 2015)

3. Vijverberg SJ, Hilvering B, Raaijmakers JA, et al., Clinical utility of asthma biomarkers: from bench to bedside, Biologics, 2013;7:199-210

4. Pavord ID, Eosinophilic phenotypes of airway disease, Ann Am Thorac Soc, 2013;10(Suppl.):S143-9.

5. Barnes PJ, New concepts in the pathogenesis of bronchial hyperresponsiveness and asthma, J Allergy Clin Immunol 1989;83:1013-26.

6. Fireman E, Toledano B, Buchner N, et al., Simplified detection of eosinophils in induced sputum, Inflamm Res, 2011;60:745-50.

7. Thomsen $\mathrm{M}$, Ingebrigtsen $\mathrm{TS}$, Marott JL, et al., Inflammatory biomarkers and exacerbations in chronic obstructive pulmonary disease, JAMA, 2013;309:2353-61.

8. Green RH, Brightling CE, Mckenna S, et al., Asthma exacerbations and sputum eosinophil counts: a randomised controlled trial, Lancet, 2002:360:1715-21.

9. Fleming L, Tsartsali L, Wilson $\mathrm{N}$, et al., Sputum inflammatory phenotypes are not stable in children with asthma, Thorax, 2012;67:675-81.

10. Sun XW, Gu SY, Li QY, et al., Pulmonary function parameters in high-resolution computed tomography phenotypes of chronic obstructive pulmonary disease, Am J Med Sci, 2015;349:228-33.

11. Miravitlles M, Calle M, Soler-Cataluña JJ, Fenotipos clínicos de la EPOC, Identificación, definición e implicaciones para las guías de tratamiento Arch Bronconeumol, 2012:48:86-98.

12. Han MK, Agusti A, Calverley PM, et al., Chronic obstructive pulmonary disease phenotypes, Am J Respir Crit Care Med, 2010;182:598-604

13. Vos T, Global, regional, and national incidence, prevalence, and years lived with disability for 301 acute and chronic diseases and injuries in 188 countries, 1990-2013: a systematic analysis for the Global Burden of Disease Study 2013, Lancet, 2015:386:743-800.

14. Bousquet J, Khaltaev NG, Cruz AA, Global Surveillance, Prevention and Control of Chronic Respiratory Diseases: A Comprehensive Approach, Geneva: World Health Organization, 2007.

15. WHO, WHO methods and data sources for global burden of disease estimates 2000-2011, Geneva: Department of Health Statistics and Information Systems, 2013. Available at: http://www.who.int/healthinfo/statistics/ GlobalDALYmethods_2000_2011.pdf (accessed on September 1, 2015)

16. Bonilla S, Kehl S, Kwong KY, et al., School absenteeism in children with asthma in a Los Angeles inner city school, J Pediatr, 2005;147:802-6.

17. Amre DK, Infante-Rivard C, Gautrin D, Malo J, Socioeconomic status and utilization of health care services among asthmatic children, J Asthma, 2002;39:625-31.

18. Lodha R, Puranik M, Kattal N, Kabra SK, Social and economic impact of childhood asthma, Indian J Pediatr. 2003:40:874-9.

19. Kinsella KG, Wan H, An Aging World: 2008, Washington DC: US
Dept. of Commerce, Economics and Statistics Administration US. Census Bureau; 2009. Available at: https://www.census.gov/ prod/2009pubs/p95-09-1.pdf (accessed on September 1, 2015)

20. Gibson GJ, Loddenkemper R, Lundback B, Sibille Y, Respiratory health and disease in Europe: the new European Lung White Book, Eur Respir J, 2013;42:559-63.

21. American Thoracic Society-Bronchoalveolar Lavage, n.d. Available at: http //wwwthoracic.org/professionals/clinicalresources/critical-care/clinical-education/critical-careprocedures/bronchoalveolar-lavage.php\#prep (accessed on September 1, 2015)

22. Moschino L, Zanconato S, Bozzetto S, et al., Childhood asthma biomarkers: present knowledge and future steps, Paediatr Respir Rev, 2015;16:205-12.

23. Fauci AS, Harrison TR, Harrison's Principles of Internal Medicine New York: McGraw-Hill, Medical Publishing Division, 2008:1667.

24. Pizzichini M, Popov T, Efthimiadis A, et al., Spontaneous and induced sputum to measure indices of airway inflammation in asthma, Am J Respir Crit Care Med, 1996;154:866-9.

25. Djukanovic R, Sterk P, Fahy J, Hargreave F, Standardised methodology of sputum induction and processing, Eur Respir J, 2002;20(Suppl. 37):1s-2s.

26. Fireman E, Bliznuk D, Schwarz Y, et al., Biological monitoring of particulate matter accumulated in the lungs of urban asthmatic children in the Tel-Aviv area, Int Arch Occup Environ Health, 2015;88:443-53.

27. Macedo P, Hew M, Torrego A, et al., Inflammatory biomarkers in airways of patients with severe asthma compared with nonsevere asthma, Clin Exp Allergy, 2009;39:1668-76.

28. Lougheed M, Lemiere C, Ducharme F, Canadian Thoracic Society 2012 guideline update: diagnosis and management of asthma in preschoolers, children and adults, Can Respir J, 2012:19:127-64.

29. Shaw J, Vaughan A, Dent A, Biomarkers of progression of chronic obstructive pulmonary disease (COPD), J Thorac Dis, 2014;6:1532-47.

30. Fireman E, Lerman $Y$, Stark M, et al., Detection of occult lung impairment in welders by induced sputum particles and breath oxidation, Am J Ind Med, 2008:51:503-11.

31. Lerman $Y$ Segal B, Rochvarger $M$, et al., Induced-sputum particle size distribution and pulmonary function in foundry workers, Arch Environ Health, 2003:58:565-71.

32. Fireman $E$, Lerman $Y$, Ganor $E$, et al., Induced sputum assessment in New York City firefighters exposed to World Trade Center dust, Environ Health Perspect, 2004;112:1564-9.

33. Wright RJ, Kelly BJ, Programming of respiratory health in childhood: influence of outdoor air pollution, Curr Opin Pediatr 2013;25:232-9.

34. Fireman E, Bliznuk D, Schwarz Y, et al., Biological monitoring of particulate matter accumulated in the lungs of urban asthmatic children in the Tel-Aviv area. Int Arch Occup Environ Health 2015;88:443-53

35. Erzurum SC, Gaston BM, Biomarkers in asthma, Clin Chest Med 2012;33:459-71.

36. Fireman E, Toledano B, Buchner N, et al., Simplified detection of eosinophils in induced sputum, Inflamm Res, 2011:60:45-750.

37. Nathan $C$, Xie $Q$, Nitric oxide synthases: roles, tolls, and controls, Cell, 1994:78:915-8.
38. Kharitonov $S$, Yates $D$, Robbins R, et al., Increased nitric oxide in exhaled air of asthmatic patients, Lancet, 1994;343:33-5

39. American Thoracic Society, European Respiratory Society, ATS/ ERS recommendations for standardized procedures for the online and offline measurement of exhaled lower respiratory nitric oxide and nasal nitric oxide, Am J Respir Crit Care Med 2005:171:912-30

40. Berry MA, Shaw DE, Green RH, et al., The use of exhaled nitric oxide concentration to identify eosinophilic airway inflammation: an observational study in adults with asthma, Clin Exp Allergy, 2005;35:1175-9.

41. Cowan D, Taylor D, Peterson L, et al., Biomarker-based asthma phenotypes of corticosteroid response, J Allergy Clin Immunol, 2015:135:877-83

42. Van Der Valk RJ, Baraldi E, Stern G, et al., Daily exhaled nitric oxide measurements and asthma exacerbations in children, Allergy, 2011;67:265-71.

43. Angelis $\mathrm{N}$, Porpodis $\mathrm{K}$, Zarogoulidis P, Airway inflammation in chronic obstructive pulmonary disease, I Thorac Dis, 2014;6:167-72.

44. Gelb AF, Barnes PJ, George SC, et al., Review of exhaled nitric oxide in chronic obstructive pulmonary disease, I Breath Res, 2012;6:047101.

45. Horvath I, Hunt J, Barnes PJ, et al., Exhaled breath condensate: methodological recommendations and unresolved questions, Eur Respir J, 2005;26:523-48.

46. Koczulla R, Dragonieri S, Schot R, et al., Comparison of exhaled breath condensate $\mathrm{pH}$ using two commercially available devices in healthy controls, asthma and COPD patients, Respir Res, 2009;10:78.

47. Konstantinidi EM, Lappas AS, Tzortzi AS, Behrakis PK, Exhaled breath condensate: technical and diagnostic aspects, Scientific World Journal, 2015:2015:1-25

48. Benor S, Alcalay Y, Domany K, et al., Ultrafine particle content in exhaled breath condensate in airways of asthmatic children, J Breath Res, 2015;9:026001

49. Leung TF, KO FW, Wong GW, Recent advances in asthma biomarker research, Ther Adv Respir Dis, 2013:7:297-308.

50. Lee JS, Shin JH, Hwang J, et al., Malondialdehyde and 3nitrotyrosine in exhaled breath condensate in retired elderly coal miners with chronic obstructive pulmonary disease, Saf Health Work, 2014:5:91-6.

51. Loukides S, Kontogianni K, Hillas G, Horvath I, Exhaled breath condensate in asthma: from bench to bedside, Curr Med Chem 2011;18:1432-43

52. Jia G, Erickson RW, Choy DF, et al., Periostin is a systemic biomarker of eosinophilic airway inflammation in asthmatic patients, J Allergy Clin Immunol, 2012:130:647-54.

53. Moffatt MF, Gut IG, Demenais F, et al., GABRIEL Consortium, A large-scale, consortium-based genomewide association study of asthma, N Engl J Med, 2010;363:1211-21.

54. Adamko DJ, Sykes BD, Rowe BH, The metabolomics of asthma: novel diagnostic potential, Chest, 2012;141:1295-302.

55. Severe Asthma Research Network (SARP). Available at: http:// www.severeasthma.org/ (accessed on September 1, 2015)

56. Home | U-BIOPRED. Available at: http://www.europeanlung.org/ en/projects-and-research/projects/u-biopred/home (accessed on September 1, 2015) 